\title{
Current Status of Phytoplasmas and their Related Diseases in Korea
}

\author{
Hee-Young Jung*, Nang Kyu Kyu Win and Young-Hwan Kim \\ School of Applied Biosiences, Kyungpook National University, Daegu 702-701, Korea \\ (Received on April 21, 2012; Revised on May 29, 2012; Accepted on May 29, 2012)
}

\begin{abstract}
Phytoplasmas have been associated with more than 46 plant species in Korea. Several vegetables, ornamentals, fruit trees and other crop species are affected by phytoplasma diseases. Six $16 \mathrm{Sr}$ groups of phytoplasmas have been identified and these phytoplasmas are associated with 63 phytoplasma diseases. Aster yellows phytoplasmas are the most prevalent group and has been associated with more than 25 diseases in Korea. Jujube witches' broom, paulownia witches' broom and mulberry dwarf diseases cause economic losses to host trees throughout the country. So far, Korean phytoplasmas belong to six species of ' $C$ andidatus Phytoplasma'; ' $\mathrm{Ca}$. P. asteris', 'Ca. P. pruni*', 'Ca. P. ziziphi', 'Ca. P. trifolii', 'Ca. P. solani*' and ' $\mathrm{Ca}$. P. castaneae'. The diseases are distributed throughout the country and most of them were observed in Gyeongbuk and Chonbuk provinces. At least four insect vectors; Cyrtopeltis tenuis, Hishimonus sellatus, Macrosteles striifrons and Ophiola flavopicta have been identified for phytoplasma transmission.
\end{abstract}

Keywords : 'Candidatus Phytoplasma' species, classification, identification

Phytoplasmas, formerly termed mycoplasma-like organisms, were discovered by a group of Japanese scientists in 1967 (Doi et al.). They are minute, wall-less pleomorphic prokaryotes located in sieve elements of the phloem of many plant species. Phytoplasmas severely affect herbaceous and woody plants exhibiting symptoms of virescence/phyllody, sterility of flowers, witches' broom, abnormal inter-node elongation, and generalized stunting. Nowadays, phytoplasma diseases are the primary limiting factors for many important crops all over the world (Bertaccini, 2007). In Korea, jujube witches' broom, mulberry dwarf, paulownia witches' broom, sumac witches' broom and chestnut witches' broom diseases cause severe losses to trees throughout the country. The Korean National Quarantine Service listed 20 phytoplasma diseases to prohibit and regulate including grapevine flavescence dorée, pear decline, potato stolbur,

\footnotetext{
*Corresponding author.

Phone) +82-53-950-5760, FAX) +82-53-950-6758

E-mail) heeyoung@knu.ac.kr
}

etc. (http://www.nqps.go.kr). Generally, identification and classification on phytoplasma diseases mainly rely on the 16S rRNA gene sequences and about 63 phytoplasma diseases have been reported. Nowadays, climatic changes are of concern to many researchers because warm weather could favor the long survival of insect vectors and also the entry of new insect vectors for phytoplasma transmission and distribution. Hence, data information on native phytoplasmas and their associated host plants in Korea is important for quarantine services. For such purpose, we had collected all available publications of phytoplasma diseases identified by electron microscopic (EM) and fluorescence microscope (FM) observation, and DNA-based classification to date. This review presents the phytoplasma diseases distributed in Korea, the associated hosts and phylogenetic status of the identified phytoplasmas.

\section{Phytoplasma detection and classification}

Before 1973, jujube witches' broom (JWB) and other diseases such as paulownia witches' broom (PaWB), mulberry dwarf(MD), and black locust witches' broom (BloWB) had been considered as viral diseases but the causal agents for those diseases had not been identified. In 1973, Yi et al. firstly reported the association of mycoplasma-like bodies with witches' broom affected jujube trees by electron microscope (EM), and Professor So In Yung (1973) identified sweet potato witches' broom by graft transmission, antibiotic reactions and EM. Then, Kim (1980) reported that witches' broom of Rhus javanica (SuWB) was caused by mycoplasma-like organisms (MLO) using EM. In 1989, Chai and Kim found MLO association with Ligustrum ovalifolium witches' broom by FM observation.

In the 1990s, molecular tools such as PCR/RFLP and nested-PCR of the conserved phytoplasma 16S rRNA gene were developed (Gundersen et al., 1996; Lee et al., 1993; Namba et al., 1993). Total DNAs were extracted from infected samples and phytoplasmas were detected by using the 16S rRNA specific primers and restriction enzyme digestion. In this way, phytoplasmas associated with paulownia, goldenrod and lilac plants were firstly documented by Lee et al. (1996). Then, Han and coworkers worked 
extensively on JWB phytoplasma and proved that it was genetically distinct from sesame phyllody, PaWB, MD, SuWB and chestnut little leaf (CLL) phytoplasmas based on the results of restriction digestion patterns of the $16 \mathrm{~S}$ rRNA gene primed by SN910601/SN910502 or R16F2/R2 (Han et al., 1997, 2001). In 1998, Han reported the presence of two phytoplasma groups in Korean trees; group I included CLL, PaWB, SuWB and MD, whereas group II included JWB phytoplasma. Chung and coworkers identified chrysanthemum phytoplasmas as aster yellows (AY) phytoplasmas by RFLP comparison to AY phytoplasmas from periwinkle (Chung et al., 2001). Also in 2003, Jung differentiated eight Korean phytoplasmas into three 16S-groups and four subgroups as aster yellows (AY) subgroup in AY 16S-group, chestnut witches' broom (CnWB) subgroup in CnWB 16S-group, and clover proliferation subgroup and JWB subgroup in Elm yellows (EY) 16S-group based on rrnA sequences of these phytoplasmas. Lee (2004) described 52 phytoplasma diseases in Korea and classified the phytoplasmas into six 'Candidatus Phytoplasma' species by analysis of the 16S rRNA gene sequences. Recently, Chung et al. (2011) described seven widespread phytoplasma diseases of floricultural crops in Korea which are associated with aster yellows, stolbur and X-disease phytoplasmas.

So far, 63 phytoplasma diseases have been reported in Korea including the quarantine important phytoplasmas. For simultaneous detection of quarantine phytoplasmas, multiplex PCR assays had been developed firstly by Kim et al. (2011) for easily detection of 10 ' $\mathrm{Ca}$. Phytoplasma' species and the phytoplasmas' association could be recognized by the specific band patterns within a short time. Aster yellows phytoplasmas group is the most prevalent group in Korea and strains generally recognized as members of AY group have not yet been studied for finer differentiation such as subgroups. To reveal the diversity among AY group phytoplasmas, Win et al. studied nine Korean AY phytoplasmas by the 16S rRNA gene sequence and RFLP analysis, and also based on interoperons heterogeneity. From that study, at least two subgroups, 16SrI-B and $16 \mathrm{SrI}-\mathrm{D}$, were present. In addition, diversity was demonstrated among 16SrI-B phytoplasmas by the presence of interoperons heterogeneity. These phytoplasmas were reclassified into three subgroups such as $16 \mathrm{SrI}-\mathrm{B}(\mathrm{a})$ if the phytoplasma possesses the identical interoperons and $16 \mathrm{SrI}-\mathrm{B}(\mathrm{b})$ or $16 \mathrm{SrI}-\mathrm{B}(\mathrm{c})$ if the phytoplasma have interoperon heterogeneity. Other conserved genes such as ribosomal protein $(r p)$ gene and elongation factor $(t u f)$ gene have been used to differentiate the AY phytoplasmas in that study (Win et al., 2012). Similarly, other AY phytoplasmas should be reclassified to the appropriate subgroups.

\section{Host plants}

Phytoplasmas are found associated with disorders of several plant species including vegetables, ornamentals, fruit trees

Table 1. Plant hosts and associated phytoplasma diseases, classification of the associated phytoplasmas as 'Candidatus Phytoplasma' species based on molecular analysis, and their geographical occurrence in Korea

\begin{tabular}{|c|c|c|c|c|c|c|c|}
\hline Plant host & Associated disease & $\begin{array}{c}\text { 'Ca. } \\
\text { Phytoplasma' }\end{array}$ & $\begin{array}{l}\text { Phytoplas- } \\
\text { ma group }\end{array}$ & $\begin{array}{l}\text { Evidence of } \\
\text { identity }^{\mathrm{a}}\end{array}$ & $\begin{array}{l}\text { Accession } \\
\text { number }\end{array}$ & $\begin{array}{l}\text { Region } \\
\text { (province) }\end{array}$ & $\begin{array}{l}\text { Selected } \\
\text { references }\end{array}$ \\
\hline \multicolumn{8}{|l|}{ Amaryllidaceae } \\
\hline Allium cepa (Onion) & $\begin{array}{l}\text { Onion yellow dwarf } \\
\text { (OYD) }\end{array}$ & 'Ca. P. asteris' & $\mathrm{AY} / \mathrm{AY}$ & SA & AB292849 & $\begin{array}{l}\text { Gangwon, } \\
\text { Gyeongnam }\end{array}$ & $\begin{array}{l}\text { Jung and Kim, } \\
2008(\mathrm{~GB})\end{array}$ \\
\hline \multicolumn{8}{|l|}{ Anacardiaceae } \\
\hline Rhus javanica (Sumac) & $\begin{array}{l}\text { Sumac witches' broom } \\
\text { (SuWB) }\end{array}$ & 'Ca. P. asteris' & AY/AY & SA & AB693125 & Chonbuk, Gyeongbuk & $\begin{array}{l}\text { Lee et al., } 2012 \\
\text { (GB) }\end{array}$ \\
\hline \multicolumn{8}{|l|}{ Apiaceae } \\
\hline $\begin{array}{l}\text { Oenanthe javanica } \\
\text { (Water dropwort) }\end{array}$ & $\begin{array}{l}\text { Water dropwort witches' } \\
\text { broom (WDWB) }\end{array}$ & 'Ca. P. asteris' & AY/AY & RFLP, SA & AB078436 & $\begin{array}{l}\text { Chonnam, Gyeong- } \\
\text { buk, Gyeongnam }\end{array}$ & $\begin{array}{l}\text { Woo et al., 2001; } \\
\text { Jung et al., 2002b }\end{array}$ \\
\hline $\begin{array}{l}\text { Bupleurum falcatum } \\
\text { (Hare's ear) }\end{array}$ & $\begin{array}{l}\text { Hare's ear yellow dwarf } \\
\text { (HEYD) }\end{array}$ & 'Ca. P. asteris' & $\mathrm{AY} / \mathrm{AY}$ & SA & AB693129 & $\begin{array}{l}\text { Chonbuk, Gyeongbuk, } \\
\text { Gyeongnam }\end{array}$ & $\begin{array}{l}\text { Choi et al., 1985; } \\
\text { 'Lee et al., } 2012 \\
\text { (GB) }\end{array}$ \\
\hline $\begin{array}{l}\text { Cnidium officinale } \\
\text { (Cnidii rhizome) }\end{array}$ & $\begin{array}{l}\text { Cnidium witches' } \\
\text { broom (CdWB) }\end{array}$ & $\begin{array}{l}\text { 'Ca. } \mathrm{P} \text {, } \\
\text { pruni*' }\end{array}$ & WX/WX & $\begin{array}{l}\text { EM, FM, } \\
\text { SA }\end{array}$ & NA & $\begin{array}{l}\text { Chungbuk, Gyeonggi, } \\
\text { Gyeongbuk (Ulleung) }\end{array}$ & Lee, 2004 \\
\hline $\begin{array}{l}\text { Cryptotaenia japonica } \\
\text { (Japanese hornwort) }\end{array}$ & $\begin{array}{l}\text { Hornwort witches' } \\
\text { broom (HWB) }\end{array}$ & unclassified & - & Symptom & - & Gyeongbuk (Ulleung) & Lee and Kim, 1994 \\
\hline \multicolumn{8}{|l|}{ Araliaceae } \\
\hline $\begin{array}{l}\text { Aralia cordata } \\
\text { (Japanese spikenard) }\end{array}$ & $\begin{array}{l}\text { Udo yellow dwarf } \\
\text { (UYD) }\end{array}$ & $\begin{array}{l}\text { 'Ca.P. } \\
\text { pruni*' }\end{array}$ & WX/WX & EM, SA & NA & Gyeongbuk (Ulleung) & Lee et al., 2002 \\
\hline
\end{tabular}


Table 1. Continued

\begin{tabular}{|c|c|c|c|c|c|c|c|}
\hline Plant host & Associated disease & $\begin{array}{c}\text { 'Ca. } \\
\text { Phytoplasma' }\end{array}$ & $\begin{array}{l}\text { Phytoplas- } \\
\text { ma group }\end{array}$ & $\begin{array}{l}\text { Evidence of } \\
\text { identity }^{\mathrm{a}}\end{array}$ & $\begin{array}{c}\text { Accession } \\
\text { number }\end{array}$ & $\begin{array}{c}\text { Region } \\
\text { (province) }\end{array}$ & $\begin{array}{l}\text { Selected } \\
\text { references }\end{array}$ \\
\hline \multicolumn{8}{|l|}{ Asteraceae } \\
\hline \multirow{2}{*}{$\begin{array}{l}\text { Dendranthema } \\
\text { grandiflorum } \\
\text { (Chrysanthemum) }\end{array}$} & $\begin{array}{l}\text { Chrysanthemum yellow } \\
\text { dwarf(ChYD) }\end{array}$ & 'Ca. P. asteris' & $\mathrm{AY} / \mathrm{AY}$ & RFLP, SA & AY241706 & Gyeonggi & \multirow{2}{*}{$\begin{array}{l}\text { Chung and Kim, } \\
2005\end{array}$} \\
\hline & $\begin{array}{l}\text { Chrysanthemum witches' } \\
\text { broom (ChWB) }\end{array}$ & $\begin{array}{l}\text { 'Ca. P. } \\
\text { solani'' }\end{array}$ & STOL & RFLP, SA & AY169308 & Gyeongnam & \\
\hline $\begin{array}{l}\text { Lactuca sativa } \\
\text { (Lettuce) }\end{array}$ & Lettuce proliferation- $\mathrm{K}$ & 'Ca. P. asteris' & AY & SA & EF489024 & Gyeonggi & Chung et al., 2007 \\
\hline $\begin{array}{l}\text { Solidago virgaurea } \\
\text { (Goldenrod) }\end{array}$ & $\begin{array}{l}\text { Korean goldenrod } \\
\text { witches' broom (GrWB) }\end{array}$ & $\begin{array}{l}\text { 'Ca. P. } \\
\text { pruni*' }\end{array}$ & WX/WX & $\begin{array}{l}\text { FM, EM, } \\
\text { RFLP }\end{array}$ & - & Gyeongbuk (Ulleung) & Lee et al., 1996 \\
\hline $\begin{array}{l}\text { Aster glehni var. } \\
\text { hondoensis }\end{array}$ & $\begin{array}{l}\text { Glehni aster yellow } \\
\text { dwarf (GAYD) }\end{array}$ & $\begin{array}{l}\text { 'Ca. P. } \\
\text { pruni*' }\end{array}$ & WX/WX & FM, SA & NA & Gyeongbuk (Ulleung) & Jung and Lee, 2004 \\
\hline Aster ciliosus & $\begin{array}{l}\text { Ciliosus aster witches' } \\
\text { broom (CAWB) }\end{array}$ & $\begin{array}{l}\text { 'Ca. P. } \\
\text { pruni*' }\end{array}$ & WX/WX & FM, SA & NA & Gyeongbuk & Lee, 2004 \\
\hline $\begin{array}{l}\text { Liatris spicata } \\
\text { (Dense blazing star) }\end{array}$ & $\begin{array}{l}\text { Liatris witches' broom } \\
\text { (LiWB) }\end{array}$ & unclassified & - & Symptom & - & Gangwon & Hahm et al., 1998 \\
\hline \multicolumn{8}{|l|}{ Buxaceae } \\
\hline $\begin{array}{l}\text { Pachysandra terminalis } \\
\text { (Japanese spurge) }\end{array}$ & $\begin{array}{l}\text { Japanese spurge } \\
\text { yellows (JSY) }\end{array}$ & 'Ca. P. asteris' & AY/16SrI-B & RFLP, SA & AB551736 & Daegu & Back et al., 2010 \\
\hline \multicolumn{8}{|l|}{ Convolvulaceae } \\
\hline $\begin{array}{l}\text { Ipomoea batatas } \\
\text { (Sweet potato) }\end{array}$ & $\begin{array}{l}\text { Sweet potato witches' } \\
\text { broom }\end{array}$ & unclassified & - & EM & - & Chonbuk & So, 1973 \\
\hline \multicolumn{8}{|l|}{ Ericaceae } \\
\hline $\begin{array}{l}\text { Rhododendron sp. } \\
\text { (Azalea) }\end{array}$ & $\begin{array}{l}\text { Azalea witches' broom } \\
\text { (AWB) }\end{array}$ & 'Ca. P. asteris' & AY & SA & AB444716 & Chonbuk & Han, 2008 (GB) \\
\hline \multicolumn{8}{|l|}{ Euphorbiaceae } \\
\hline \multirow{2}{*}{$\begin{array}{l}\text { Euphorbia pulcherrima } \\
\text { (Poinsettia) }\end{array}$} & $\begin{array}{l}\text { Poinsettia stem flat } \\
\text { (PoiSF) }\end{array}$ & 'Ca. P. pruni*' & 16SrIII-H & SA & GU461275 & Gyeonggi & \multirow{2}{*}{$\begin{array}{l}\text { Chung and Choi, } \\
\text {-2010 }\end{array}$} \\
\hline & $\begin{array}{l}\text { Poinsettia branch-induc- } \\
\text { ing (PoiBI) }\end{array}$ & $\begin{array}{l}\text { 'Ca. P. } \\
\text { pruni*' }\end{array}$ & $16 \mathrm{SrIII}-\mathrm{H}$ & SA & GU461277 & Gyeonggi & \\
\hline \multicolumn{8}{|l|}{ Fabaceae } \\
\hline $\begin{array}{l}\text { Robinia pseudoacacia } \\
\text { (Black locust) }\end{array}$ & $\begin{array}{l}\text { Locust witches' broom } \\
\text { (BLWB) }\end{array}$ & 'Ca. P. asteris' & $16 \mathrm{SrI}$ & SA & NA & Gangwon, Chonbuk & Han, 2007 \\
\hline Trifolium repens (Clover) & $\begin{array}{l}\text { Clover yellow dwarf } \\
\text { (CYD) }\end{array}$ & unclassified & - & EM & - & Gyeongbuk & Chang, 1981 \\
\hline $\begin{array}{l}\text { Lespedeza cyrtobotrya } \\
\text { (Leafy lespedeza) }\end{array}$ & $\begin{array}{l}\text { Leafy lespedeza witches’ } \\
\text { broom (LLWB) }\end{array}$ & 'Ca. P. trifolii' & - & RFLP, SA & AB279597 & Gangwon & $\begin{array}{l}\text { Kim and Jung, } \\
2007\end{array}$ \\
\hline \multicolumn{8}{|l|}{ Fagaceae } \\
\hline \multirow{2}{*}{$\begin{array}{l}\text { Castanea crenata } \\
\text { (Japanese chestnut) }\end{array}$} & Chestnut little leaf(CLL) & 'Ca. P. asteris' & AY & RFLP & - & Chonbuk, Chonnam & Han and Cha, 2001 \\
\hline & $\begin{array}{l}\text { Chestnut witches' broom } \\
\text { (CnWB) }\end{array}$ & $\begin{array}{l}\text { 'Ca. P. casta- } \\
\text { neae' }\end{array}$ & CnWB & SA & AB054986 & $\begin{array}{l}\text { Chonbuk, } \\
\text { Gyeongnam }\end{array}$ & Jung et al., 2002a \\
\hline $\begin{array}{l}\text { Quercus acutissima } \\
\text { (Sawtooth oak) }\end{array}$ & $\begin{array}{l}\text { Chestnut oak witches' } \\
\text { broom (COWB) }\end{array}$ & unclassified & - & Symptom & - & Gyeongnam & Lee and Kim, 1994 \\
\hline $\begin{array}{l}\text { Castanopsis cuspidata } \\
\text { (Japanese chinquapin) }\end{array}$ & $\begin{array}{l}\text { Castanopsis witches' } \\
\text { broom (CasWB) }\end{array}$ & unclassified & - & EM, FM & - & Gyeongnam, Jeju & $\begin{array}{l}\text { Lee et al., } \\
\text { unpublished }\end{array}$ \\
\hline \multicolumn{8}{|l|}{ Gentianaceae } \\
\hline $\begin{array}{l}\text { Gentiana scabra } \\
\text { (Japanese gentian) }\end{array}$ & $\begin{array}{l}\text { Gentian witches' broom } \\
\text { (GWB) }\end{array}$ & unclassified & - & FM, PCR & - & Gyeongbuk & Lee, 2004 \\
\hline
\end{tabular}


Table 1. Continued

\begin{tabular}{|c|c|c|c|c|c|c|c|}
\hline Plant host & Associated disease & $\begin{array}{c}\text { 'Ca. } \\
\text { Phytoplasma' }\end{array}$ & $\begin{array}{l}\text { Phytoplas- } \\
\text { ma group }\end{array}$ & $\begin{array}{l}\text { Evidence of } \\
\text { identity }^{\mathrm{a}}\end{array}$ & $\begin{array}{l}\text { Accession } \\
\text { number }\end{array}$ & $\begin{array}{l}\text { Region } \\
\text { (province) }\end{array}$ & $\begin{array}{l}\text { Selected } \\
\text { references }\end{array}$ \\
\hline \multicolumn{8}{|l|}{ Liliaceae } \\
\hline Lilium longiflorum (Lily) & $\begin{array}{l}\text { Lily flattened stem } \\
\text { (Ph-lily) }\end{array}$ & $\begin{array}{l}\text { 'Ca.P. } \\
\text { solani*' }\end{array}$ & $16 \mathrm{SrXIII}-\mathrm{A}$ & RFLP, SA & AY169309 & Gyeonggi, Jeju & $\begin{array}{l}\text { Chung and Jeong, } \\
2003\end{array}$ \\
\hline \multicolumn{8}{|l|}{ Moraceae } \\
\hline Morus alba (Mulberry) & Mulberry dwarf(MD) & 'Ca. P. asteris' & $16 \mathrm{SrI}$ & EM, SA & AY075038 & Chonbuk, Gyeongbuk & kHan and Cha, 2002 \\
\hline \multicolumn{8}{|l|}{ Oleaceae } \\
\hline $\begin{array}{l}\text { Chionanthus retusus } \\
\text { (Snow-flower fringe) }\end{array}$ & $\begin{array}{l}\text { Snow-flower fringe tree } \\
\text { dwarf(SFTD) }\end{array}$ & $\begin{array}{l}\text { 'Ca. P. ast- } \\
\text { eris' }\end{array}$ & AY/AY & RFLP, SA & NA & Chonbuk & Lee, 2004 \\
\hline $\begin{array}{l}\text { Fraxinus sieboldiana } \\
\text { (Japanese Ash) }\end{array}$ & $\begin{array}{l}\text { Angustata ash witches' } \\
\text { broom (AAshWB) }\end{array}$ & 'Ca. P. asteris' & $\mathrm{AY} / \mathrm{AY}$ & RFLP,SA & AB693130 & Chonbuk & $\begin{array}{l}\text { Yea and Lee, 2002; } \\
\text { Lee et al., } 2012 \\
\text { (GB) }\end{array}$ \\
\hline F. rhynchophylla (Ash) & $\begin{array}{l}\text { Ash witches' broom } \\
\text { (AshWB) }\end{array}$ & 'Ca. P. asteris' & $\mathrm{AY} / \mathrm{AY}$ & SA & AY566302 & Chonbuk & Han, 2005 \\
\hline Forsythia sp. (Forsythia) & $\begin{array}{l}\text { Forsythia witches' } \\
\text { broom (FWB) }\end{array}$ & unclassified & - & RFLP, SH & - & Gyeonggi & $\begin{array}{l}\text { Han et al., } \\
\text { unpublished }\end{array}$ \\
\hline $\begin{array}{l}\text { Ligustrum japonicum } \\
\text { (Wax-leaf privet) }\end{array}$ & $\begin{array}{l}\text { Wax-leaf privet witches' } \\
\text { broom (WPrWB) }\end{array}$ & $\begin{array}{l}\text { 'Ca. P. ziz- } \\
\text { iphi' }\end{array}$ & EY/JWB & RFLP SA & AB293422 & Gyeongbuk, Jeju & $\begin{array}{l}\text { Lee, 2004; Jung } \\
\text { and Kim, } 2008 \\
\text { (GB) }\end{array}$ \\
\hline $\begin{array}{l}\text { L. lucidum } \\
\text { (Chinese privet) }\end{array}$ & $\begin{array}{l}\text { Chinese privet witches' } \\
\text { broom (CPrWB) }\end{array}$ & $\begin{array}{l}\text { 'Ca. P. } \\
\text { zizyphi' }\end{array}$ & $\mathrm{EY} / \mathrm{JWB}$ & SA & NA & Jeju & Lee, 2004 \\
\hline $\begin{array}{l}\text { L. obtusifolium } \\
\text { (Ovtusifolium privet) }\end{array}$ & $\begin{array}{l}\text { Privet witches' broom } \\
\text { (PrWB) }\end{array}$ & $\begin{array}{l}\text { 'Ca. P. ziz- } \\
\text { iphi' }\end{array}$ & $\mathrm{EY} / \mathrm{JWB}$ & EM, SA & NA & Gyeongbuk & Lee, 2004 \\
\hline $\begin{array}{l}\text { L. ibota } \\
\text { (Microphylla privet) }\end{array}$ & $\begin{array}{l}\text { Microphylla privet } \\
\text { witches' broom } \\
\text { (MPWB) }\end{array}$ & unclassified & - & EM & - & Chonbuk & $\begin{array}{l}\text { Chai et al., } \\
\text { unpublished }\end{array}$ \\
\hline $\begin{array}{l}\text { L. ovalifolium } \\
\text { (Oval-Leaved privet) }\end{array}$ & $\begin{array}{l}\text { Ovalifola privet witches' } \\
\text { broom }\end{array}$ & unclassified & - & EM, FM & - & Chonbuk, Chungnam & $\begin{array}{l}\text { Chai and Kim, } \\
1989\end{array}$ \\
\hline $\begin{array}{l}\text { L. foliosum } \\
\text { (Korean privet) }\end{array}$ & $\begin{array}{l}\text { Korean privet witches' } \\
\text { broom (KprWB) }\end{array}$ & $\begin{array}{l}\text { 'Ca. P. ziz- } \\
\text { iphi' }\end{array}$ & EY/JWB & SA & NA & Gyeongbuk (Ulleung) & Lee, 2004 \\
\hline $\begin{array}{l}\text { Syringa vulgaris } \\
\text { (Lilac) }\end{array}$ & $\begin{array}{l}\text { Lilac witches' broom } \\
\text { (LWB) }\end{array}$ & $\begin{array}{l}\text { 'Ca. P. ziz- } \\
\text { iphi' }\end{array}$ & - & RFLP, SA & NA & Gyeongbuk & Lee et al., 1996 \\
\hline
\end{tabular}

\section{Paulowniaceae}

\begin{tabular}{|c|c|c|c|c|c|c|}
\hline $\begin{array}{l}\text { Paulownia coreana } \\
\text { (Paulownia) }\end{array}$ & $\begin{array}{l}\text { Paulownia witches' } \\
\text { broom (PaWB) }\end{array}$ & 'Ca. P. asteris' & AY/AY & RFLP, SA & AF279271 Gyeonggi & Han et al., 2001 \\
\hline $\begin{array}{l}\text { P. tomentosa } \\
\text { (Princess tree) }\end{array}$ & $\begin{array}{l}\text { Paulownia witches' } \\
\text { broom (PaWB) }\end{array}$ & 'Ca. P. asteris' & AY/AY & RFLP, SA & AB693131 $\begin{array}{l}\text { Gyeonggi, } \\
\text { Gyeongbuk }\end{array}$ & $\begin{array}{l}\text { Han and Cha, 2001; } \\
\text { Lee et al., } 2012 \\
\text { (GB) }\end{array}$ \\
\hline
\end{tabular}

\begin{tabular}{|c|c|c|c|c|c|c|c|}
\hline Pedaliaceae & & & & & & & \\
\hline $\begin{array}{l}\text { Sesamum indicum } \\
\text { (Sesame) }\end{array}$ & $\begin{array}{l}\text { Sesame yellow dwarf } \\
\text { (SeYD) }\end{array}$ & 'Ca. P. asteris' & AY/AY & EM, SA & NA & $\begin{array}{l}\text { Gyeongbuk, Gyeong- } \\
\text { nam }\end{array}$ & Lee, 2004 \\
\hline \multicolumn{8}{|l|}{ Plantaginaceae } \\
\hline $\begin{array}{l}\text { Plantago lanceolata } \\
\text { (Ribwort plantain) }\end{array}$ & Leechwort dwarf(LwD) & $\begin{array}{l}\text { 'Ca. P. } \\
\text { pruni*', }\end{array}$ & WX/WX & SA & NA & Gyeongbuk & Lee, 2004 \\
\hline $\begin{array}{l}\text { P. asiatica } \\
\text { (Chinese plantain) }\end{array}$ & $\begin{array}{l}\text { Asian plantain witches' } \\
\text { broom (PlWB) }\end{array}$ & $\begin{array}{l}\text { 'Ca. P. } \\
\text { pruni*' }\end{array}$ & WX/WX & SA & NA & Gangwon & Lee, 2004 \\
\hline \multicolumn{8}{|l|}{ Plumbaginaceae } \\
\hline $\begin{array}{l}\text { Limonium sinuatum } \\
\text { (Statice) }\end{array}$ & $\begin{array}{l}\text { Statice witches' } \\
\text { broom(StaWB) }\end{array}$ & 'Ca. P. asteris' & AY & SA & DQ192513 & Busan, Gangwon & $\begin{array}{l}\text { Hahm et al., 1998; } \\
\text { Chung et al., } 2005\end{array}$ \\
\hline
\end{tabular}


Table 1. Continued

\begin{tabular}{|c|c|c|c|c|c|c|c|}
\hline Plant host & Associated disease & $\begin{array}{c}\text { 'Ca. } \\
\text { Phytoplasma' }\end{array}$ & $\begin{array}{l}\text { Phytoplas- } \\
\text { ma group }\end{array}$ & $\begin{array}{l}\text { Evidence of } \\
\text { identity }^{\mathrm{a}}\end{array}$ & $\begin{array}{l}\text { Accession } \\
\text { number }\end{array}$ & $\begin{array}{c}\text { Region } \\
\text { (province) }\end{array}$ & $\begin{array}{l}\text { Selected } \\
\text { references }\end{array}$ \\
\hline \multicolumn{8}{|l|}{ Poaceae } \\
\hline $\begin{array}{l}\text { Phyllostachys bambusoides } \\
\text { (Japanese timber bamboo) }\end{array}$ & $\begin{array}{l}\text { Bamboo witches' broom } \\
\text { (BWB) }\end{array}$ & 'Ca. P. asteris' & AY/AY & SA & NA & $\begin{array}{l}\text { Chonbuk, Chonnam, } \\
\text { Chungnam, Gyeong- } \\
\text { buk, Gyeongnam }\end{array}$ & Lee, 2004 \\
\hline $\begin{array}{l}\text { Phyllostachys nigra } \\
\text { (Black bamboo) }\end{array}$ & $\begin{array}{l}\text { Henonis bamboo } \\
\text { witches' broom } \\
\text { (HBWB) }\end{array}$ & 'Ca. P. asteris' & AY/AY & SA & AB242433 & $\begin{array}{l}\text { Chonnam, Chung- } \\
\text { nam Gyeongbuk, } \\
\text { Gyeongnam }\end{array}$ & Jung et al., 2006 \\
\hline $\begin{array}{l}\text { Sasa borealis } \\
\text { (Northern bamboo) }\end{array}$ & $\begin{array}{l}\text { Sasa witches' broom } \\
\text { (SaWB) }\end{array}$ & 'Ca. P. asteris' & AY/AY & SA & AB293421 & $\begin{array}{l}\text { Chonnam, } \\
\text { Gyeongnam }\end{array}$ & $\begin{array}{l}\text { Jung and Kim, } \\
2009(\mathrm{~GB})\end{array}$ \\
\hline \multicolumn{8}{|l|}{ Rhamnaceae } \\
\hline \multirow{5}{*}{$\begin{array}{l}\text { Zizyphus jujuba } \\
\text { (Jujube) }\end{array}$} & $\begin{array}{l}\text { Jujube witches' broom } \\
\text { (JWB) }\end{array}$ & $\begin{array}{l}\text { 'Ca. } \\
\text { P. ziziphi' }\end{array}$ & $\mathrm{EY} / \mathrm{JWB}$ & SA & AB052879 & $\begin{array}{l}\text { Chonbuk, Chonnam, } \\
\text { Chungbuk, Chung- } \\
\text { nam, Gangwon, } \\
\text { Gyeonggi, Gyeong- } \\
\text { buk, Gyeongnam }\end{array}$ & $\begin{array}{l}\text { Han et al., 2001; } \\
\text { Jung et al., 2003b }\end{array}$ \\
\hline & & 'Ca. P. asteris' & $16 \mathrm{SrI}$ & RFLP, SA & NA & Chonbuk & Lee et al., 2009 \\
\hline & $\begin{array}{l}\text { Jujube yellow leaf roll } \\
\text { (JYLR) }\end{array}$ & $\begin{array}{c}\text { 'Ca. } \\
\text { P. ziziphi' }\end{array}$ & $\mathrm{EY} / \mathrm{JWB}$ & SA & NA & Gyeongbuk & Lee, 2004 \\
\hline & $\begin{array}{l}\text { Wild jujube witches' } \\
\text { broom (WJWB) }\end{array}$ & $\begin{array}{c}\text { 'Ca. } \\
\text { P. ziziphi' }\end{array}$ & EY/JWB & SA & NA & Gyeongbuk & Lee, 2004 \\
\hline & $\begin{array}{l}\text { Spinosa witches' broom } \\
\text { (SJWB) }\end{array}$ & $\begin{array}{l}\text { 'Ca. } \\
\text { P. ziziphi' }\end{array}$ & EY/JWB & SA & NA & Gyeongbuk & Lee, 2004 \\
\hline $\begin{array}{l}\text { Hovenia dulcis } \\
\text { (Oriental raisin) }\end{array}$ & $\begin{array}{l}\text { Japanese raisin witches' } \\
\text { broom (JRWB) }\end{array}$ & $\begin{array}{l}\text { 'Ca. } \\
\text { P. ziziphi' }\end{array}$ & $16 \mathrm{SrV}-\mathrm{B}$ & SA & $\mathrm{AB} 442218$ & Chonbuk & $\begin{array}{l}\text { Kamala-Kannan et } \\
\text { al., } 2011\end{array}$ \\
\hline \multicolumn{8}{|l|}{ Rosaceae } \\
\hline $\begin{array}{l}\text { Rubus crataegifolius } \\
\text { (Raspberry) }\end{array}$ & $\begin{array}{l}\text { Rubus witches' broom } \\
\text { (RWB) }\end{array}$ & unclassified & - & EM & - & Gyeongbuk & Lee and Yea, 1992 \\
\hline $\begin{array}{l}\text { Crataegus pinnatifida } \\
\text { (Chinese hawthorn) }\end{array}$ & $\begin{array}{l}\text { Hawthorn witches' } \\
\text { broom (HaWB) }\end{array}$ & unclassified & - & EM, PCR & - & $\begin{array}{l}\text { Gyeongbuk, } \\
\text { Gyeongnam }\end{array}$ & Yea and Lee, 1997 \\
\hline $\begin{array}{l}\text { Prunus salicina } \\
\text { (Chinese plum) }\end{array}$ & $\begin{array}{l}\text { Plum witches' broom } \\
\text { (PluWB) }\end{array}$ & unclassified & - & Symptom & - & Gyeongbuk & Lee and Kim, 1994 \\
\hline \multicolumn{8}{|l|}{ Sapindaceae } \\
\hline $\begin{array}{l}\text { Koelreuteria paniculata } \\
\text { (Goldenrain tree) }\end{array}$ & Goldenrain stunt (GRP) & 'Ca. P. asteris' & 16SrI-B & SA & EU430729 & Chonbuk & $\begin{array}{l}\text { Kamala-Kannan et } \\
\text { al., } 2010\end{array}$ \\
\hline \multicolumn{8}{|l|}{ Solanaceae } \\
\hline Petunia (Petunia) & $\begin{array}{l}\text { Petunia flat stem } \\
\text { (PFS-K) }\end{array}$ & 'Ca. P. asteris' & AY & SA & EU267779 & Gyeonggi & $\begin{array}{l}\text { Chung and Huh, } \\
2008\end{array}$ \\
\hline $\begin{array}{l}\text { Solanum tuberosum } \\
\text { (Potato) }\end{array}$ & $\begin{array}{l}\text { Potato witches' broom } \\
\text { (PWB) }\end{array}$ & 'Ca. P. trifolii' & $\mathrm{EY} / \mathrm{CP}$ & SA & AB076404 & Gangwon, Jeju & $\begin{array}{l}\text { Hahm et al., 2001; } \\
\text { Jung et al., 2003a }\end{array}$ \\
\hline \multicolumn{8}{|l|}{ Vitaceae } \\
\hline $\begin{array}{l}\text { Ampelopsis } \\
\text { brevipedunculata } \\
\text { (Porcelain berry) }\end{array}$ & $\begin{array}{l}\text { Porcelain vine witches' } \\
\text { broom }(\mathrm{PvWB})\end{array}$ & 'Ca. P. asteris' & AY/AY & SA & AB693126 & Gyeongbuk (Ulleung) & $\begin{array}{l}\text { Jung et al., 2003c; } \\
\text { Lee et al., } 2012 \\
\text { (GB) }\end{array}$ \\
\hline $\begin{array}{l}\text { Vitis amurensis } \\
\text { (Amur grape) }\end{array}$ & $\begin{array}{l}\text { Amur grape witches' } \\
\text { broom (AGWB) }\end{array}$ & 'Ca. P. asteris' & $\mathrm{AY} / \mathrm{AY}$ & SA & NA & Gyeongbuk (Ulleung) & Lee, 2004 \\
\hline Vitis vinifera (Grape vine) & Grapevine little leave & - & $16 \mathrm{SrV}$ & RFLP, SA & NA & Gyeongbuk & $\begin{array}{l}\text { Kim et al., } \\
\text { unpublished }\end{array}$ \\
\hline
\end{tabular}

${ }^{a}$ Evidence of phytoplasma: EM, electron microscopy; FM, fluorescence microscopy; PCR, polymerase chain reaction; RFLP, RFLP patterns of PCR-amplified 16S rRNA gene; SA, sequence analysis of 16S rRNA gene; SH, Southern hybridization; NA, sequence not available; GB, GenBank data 
and other crops. A total of 46 plant species have been affected by the phytoplasmas and those plants belong to 25 plant families (Table 1). Most phytoplasmas have been identified from the plants belong to two families, the Oleaceae and the Asteraceae. The widest host ranges ( 25 plant species) have been associated with AY phytoplasmas including medicinal plants (e.g. hare's ear, mulberry), vegetables (e.g. lettuce, onion, water dropwort), ornamentals (e.g. Japanese spurge, chrysanthemum, poinsettia), shrubs (sumac, lilac) and fruit trees (jujube, chestnut) which belong to 15 plant families (Back et al., 2010; Chung and Choi, 2010; Chung and Kim, 2005; Han and Cha, 2001; Lee, 2004; Lee et al., 2009). Elm yellows phytoplasmas have been associated with 10 species of trees belonging to two families, the Oleaceae and the Rhamnaceae. Nine plant species belonging to five families have been affected by western $\mathrm{X}$ phytoplasmas. Stolbur phytoplasmas have been identified from two floral plants; chrysanthemum (Asteraceae) and lily (Liliaceae) (Chung and Jeong, 2003; Chung and Kim, 2005). Clover proliferation phytoplasma has been reported from lespedeza (Fabaceae) and potato (Solanaceae) (Jung et al., 2003a; Kim and Jung, 2007). Japanese chestnut witches' broom phytoplasma has been associated with chestnut trees (Fagaceae) (Jung et al., 2002a). Some plants such as chrysanthemum, chestnut and jujube are infected by two different phytoplasma groups. Chung and coworkers reported two groups of phytoplasmas, AY and stolbur in chrysanthemum from two geographically distinct locations, in northern and southern parts of Korea (Chung and Kim, 2005). Mixed infection of phytoplasmas (16S rDNA I and V group) on jujube witches' broom disease had been identified by Lee and coworkers (Lee et al., 2009). The mixed infection was assumed to be due to the same insect vector (Hishimonus sellatus) which is able to transmit both of these two phytoplasma groups. Natural insect vectors species have a large influence the plant host range for phytoplasma (Hogenhout et al., 2008).

\section{'Candidatus Phytoplasma' species}

The phylogenetic analysis of all available Korean phytoplasma 16S rRNA gene sequences showed that the native phytoplasmas belong to six species of ' $\mathrm{Ca}$. Phytoplasma'; 'Ca. P. asteris', 'Ca. P. ziziphi', 'Ca. P. trifolii', 'Ca. P. castaneae', ' $\mathrm{Ca}$. P. pruni*' and ' $\mathrm{Ca}$. P. solani*' ${ }^{*}$ is an incidental citation and does not constitute prior citation (Lapage et al., 1992)] (Figure 1). Jung and coworkers had firstly proposed as the novel taxon as ' $\mathrm{Ca}$. P. castaneae' for the chestnut witches' broom (AB054986) phytoplasma (Jung et al., 2002a) and ' $C a$. P. ziziphi' jujube witches' broom (AB052879) (Jung et al., 2003b) following International Research Programme for Comparative Mycoplasmology adoption of a taxonomic rule previously established for recording properties of uncultured organisms (Murray and Schleifer, 1994). The existence of ' $C a$. P. solani*' association with lily stem flattening disease was reported by Chung and Jeong (2003). Association of ' $C a$. P. pruni*' association with yellow dwarf disease of Aster glehni var. hondoensis was reported in Ulleungdo (Jung and Lee, 2004). The association of ' $\mathrm{Ca}$. P. trifolii' with witches' broom disease of leafy lespedeza was observed in Pyeongchang-gun (Kim and Jung, 2007).

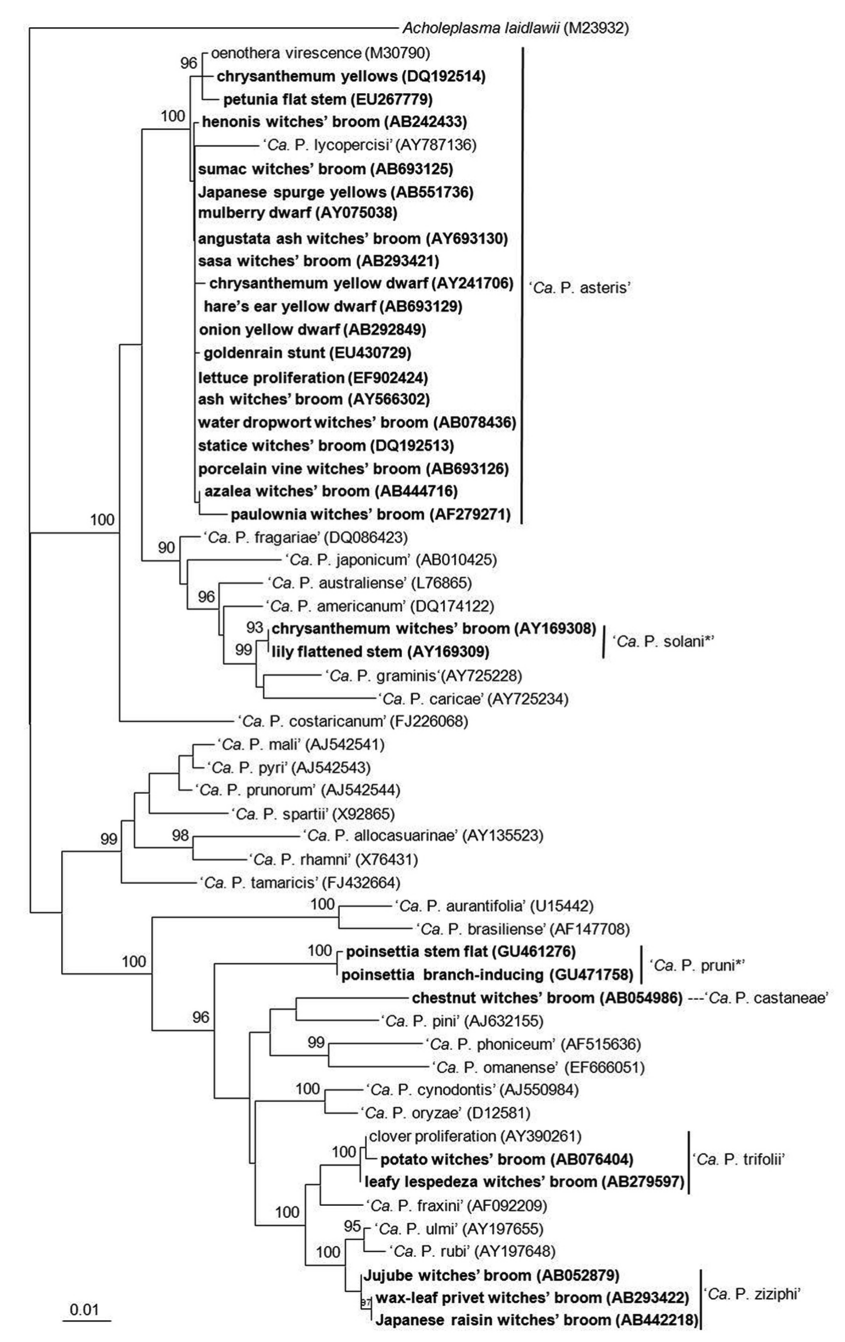

Fig. 1. Phylogenetic tree constructed by neighbor joining method of the 16S rRNA gene sequences from Korean phytoplasmas (bold font) and those of 27 previously described 'Candidatus Phytoplasma' retrieved from the Genbank. Acholeplasma laidlawii was used as an outgroup. The tree was constructed with a 100replicate bootstrap search (only values above $80 \%$ are shown) using MEGA4 software (Tamura et al., 2007). The bar represents a phylogenetic distance of $1 \%$. *According to Rule $28 \mathrm{~b}$ of the Bacteriological Code, this is an incidental citation and does not constitute prior citation (Lapage et al., 1992). 


\section{Geographic distribution and insect vectors}

In Korea, phytoplasma diseases exist in every province including Jeju and Ulleung Islands, and many diseases were reported especially from Gyeoungbuk and Chonbuk provinces. This suggests that intensive research has been conducted on phytoplasma diseases in those areas, and that more research on phytoplasmas is needed in other provinces. ' $\mathrm{Ca}$. P. asteris' and ' $\mathrm{Ca}$. P. ziziphi' associated diseases are distributed in every province, probably related to the distribution of insect vectors for those two phytoplasma species. The rhombic-marked leafhopper, Hishimonus sellatus is a specific insect vector for JWB phytoplasma (Jung et al., 2003b) and it is also able to transmit SuWB and MD phytoplasmas (La and Woo, 1980; La et al., 1984). This hopper may be the main factor in abundance of ' $\mathrm{Ca}$. P. asteris' and ' $\mathrm{Ca}$. P. ziziphi' associated diseases throughout the country. In addition, other insect vectors reported for aster yellows phytoplasma include the tobacco leaf bug, Cyrtopeltis tenuis (La and Bak, 1994); the leafhopper Empoasca sp. (Yeo et al., 1994) that can transmit PaWB; and the polyphagous leafhopper, Macrosteles striifrons (M. orientalis) that is presumed to be a vector of water dropwort witches' broom phytoplasma (Woo et al., 2001). The presence of large numbers of AY associated diseases suggests that there may be other natural vectors which are able to transmit the AY phytoplasmas. In northern and eastern parts of the country, ' $\mathrm{Ca}$. P. pruni'" associated diseases are observed and Ophiola flavopicta is the vector for one of ' $\mathrm{Ca}$. P. pruni*' associated diseases; cnidium witches' broom phytoplasmas (Lee, 1994). The diseases associated with ' $\mathrm{Ca}$. P. trifolii*' have been described from only Gangwon province and Jeju Island, and the insect vector for ' $\mathrm{Ca}$. P. trifolii'' could not yet be identified. To date, ' $C a$. P. castaneae' appears to be a host specific phytoplasma that has only been observed on chestnut trees in Gyeongnam and Chonbuk. The insects that vector chestnut witches' broom phytoplasmas are still unknown. Since many important diseases are transmitted and distributed by insect vectors, research on finding natural vectors will be very important in designing effective control measures for phytoplasma associated diseases. Research studies of phytoplasma-vector relationships or interactions are still needed in Korea.

\section{Conclusion}

This review presents the progress in differentiating and classifying the phytoplasmas reported in Korea over the last three decades. Generally, the 16S rRNA gene sequence and RFLP analysis has been widely applied in the characterization and identification of phytoplasmas. Examined total of 63 phytoplasma diseases can be attributed to one of six presumptive species ' $\mathrm{Ca}$. Phytoplasma' on the basis of $16 \mathrm{~S}$ rRNA gene sequences. Diversity of AY group phytoplasmas belonging to $16 \mathrm{SrII}-\mathrm{B}$ subgroups has been observed and these phytoplasmas have been reclassified on the basis of interoperon heterogeneity. Likewise, the second largest disease group induced by ' $\mathrm{Ca}$. P. pruni*' should be investigated to determine their phylogenetic diversity. Besides, some phytoplasma diseases which were confirmed only by symptoms or EM observation still remain to be classified at the molecular level (Table 1). Information concerning vector-phytoplasma and vector-plant relationships or interactions is necessary for improved understanding of phytoplasma-associated diseases. Although several genome projects have been started in the world in the late 1990s, only four phytoplasma genomes have been fully sequenced including those of two ' $\mathrm{Ca}$. P. asteris' strains, and strains of 'Ca. P. mali' and 'Ca. P. australiense' (Bai et al., 2006; Kube et al., 2008; Oshima et al., 2004; Tran-Nguyen et al., 2008). In our laboratory, phytoplasmas genome sequencing, especially from woody plants affected by jujube witches' broom phytoplasma, ' $\mathrm{Ca}$. P. ziziphi', is currently being conducted. Moreover, for understanding host-phytoplasma interactions, it is important to identify the function of membrane proteins or secreted proteins encoded in the phytoplasma genome. Recently, Namba and coworkers have reported one secreted protein encoded in the phytoplasma genome named as TENGU, which induces witches' broom and dwarfism (Namba, 2011). Like TENGU, other virulence factors of phytoplasmas are also challenges for researchers.

\section{Acknowledgments}

This research was supported by Kyungpook National University Research Fund, 2012.

\section{References}

Back, C. G., Chung, H. and Jung, H. Y. 2010. First report of Candidatus Phytoplasma asteris associated with yellowing and rosetting of Japanese spurge in Korea. J. Gen. Plant Pathol. 76:355-357.

Bai, X., Zhang, J., Ewing, A., Miller, S. A., Radek, A. J., Shevchenko, D. V., Tsukerman, K., Walunas, T., Lapidus, A., Campbell, J. W. and Hogenhout, S. A. 2006. Living with genome instability: the adaptation of phytoplasmas to diverse environments of their insect and plant hosts. J. Bacteriol. 188:3682-3696.

Bertaccini, A. 2007. Phytoplasmas: diversity, taxonomy, and epidemiology. Front. Biosci. 12:673-689.

Chai, J. K. and Kim, Y. H. 1989. Studies on witches' broom of Ligustrum ovalifolium Hasskari caused by mycoplasma-like organism (MLO). J. Kor. For. Soc. 78:103-118. 
Chang, M. U. 1981. Electron microscopic study of mycoplasmalike organisms in yellow- diseased plants with jujube witches' broom and clover dwarf. J. Nat. Sci. (Yeungnam Univ.) $1: 175-181$.

Choi, Y. M., Lee, S. H., Lee, E. K. and Kim, J. S. 1985. An inverstigation of undescribed witches' broom symptom disease caused by mycoplasma-like organism on Bupleurum falcatum, Cnidium officinale and Plantago asiatica in Korea. Kor. J. Mycol. 13:49-51.

Chung, B. N. and Choi, G. S. 2010. Occurrence of poinsettia stem flat disease caused by phytoplasma in Korea. Plant Dis. 94:792.

Chung, B. N., Choi, G. S., Kim, H. R. and Choi, Y. M. 2001. Identification of aster yellows phytoplasma in Dendranthema grandiflorum. Plant Pathol. J. 17:57-61.

Chung, B. N. and Huh, K. Y. 2008. Occurrence of petunia flattened stem caused by phytoplasma. Plant Pathol. J. 24:279282.

Chung, B. N., Huh, K. Y. and Jeong, M. I. 2005. First report on the witches' broom in annual statice (Limonium sinuatum) in Korea. Plant Pathol. J. 21:383-386.

Chung, B. N. and Jeong, M. I. 2003. Detection and molecular characterization of a stolbur phytoplasma in Lilium oriental hybrids. Plant Pathol. J. 19:106-110.

Chung, B. N., Jeong, M. I. and Choi, G. S. 2011. Characterization of phytoplasmal disease occurred on floricultural crops in Korea. Res. Plant Dis. 17:265-271.

Chung, B. N. and Kim, B. D. 2005. Two groups of phytoplasma from chrysanthemum (Dendranthema grandiflorum) distinguished by symptoms and 16S rRNA gene sequence in Korea. Plant Pathol. J. 21:132-136.

Chung, B. N., Kim, J. S. and Cheong, S. R. 2007. Phytoplasmaassociated shoot proliferation and leaf yellowing in lettuce. Plant Pathol. J. 23:151-154.

Doi, Y., Teranaka, M., Yora, K. and Asuyama, H. 1967. Mycoplasma or PLT grouplike microorganisms found in the phloem elements of plants infected with mulberry dwarf, potato witches' broom, aster yellows or paulownia witches' broom. Ann. Phytopathol. Soc. Jpn. 33:259-266.

Gundersen, D. E., Lee, I. M., Schaff, D. A., Harrison, N. A., Chang, C. J., Davis, R. E. and Kingsbury, D. T. 1996. Genomic diversity and differentiation among phytoplasma strains in the $16 \mathrm{~S}$ rRNA groups I (aster yellows and related phytoplasmas) and III (X-disease and related phytoplasmas). Int. J. Syst. Bacteriol. 46:64-75.

Hahm, Y. I., Kwon, M., Kim, J. S., Seo, H. W. and Ahn, J. H. 1998. Surveys on disease occurrence in major horticultural crops in Kangwon alpine areas. Korean J. Plant Pathol. 14:668-675.

Hahm, Y. I., Ryu, K. Y. and Cho, I. C. 2001. Occurrence of potato witches' broom caused by a phytoplasma in Korea. Res. Plant Dis. 7:116-119.

Han, M. S., Noh, E. W. and Yun, J. K. 1997. Occurrence of sesame phyllody disease in Korea and detection of its phytoplasma. Korean J. Plant Pathol. 13:239-243.

Han, M. S., Noh, E. W. and Yun, J. K. 2001. Differentiation of phytoplasmas infecting Zizyphus jujuba and Paulownia coreana using PCR-RFLP. Plant Pathol. J. 17:189-193.

Han, S. 1998. Studies on little leaf disease of chestnut tree (Castanea crenata Sieb. et Zucc.) in Korea. Ph.D. Thesis. Chonbuk National University. Korea. 91pp.

Han, S. 2005. Association of aster yellow phytoplasma with witches' broom disease of ash (Fraxinus rhynchophylla Hence) in Korea. J. Kor. For. Soc. 94:103-107.

Han, S. 2007. Detection of "Candidatus Phytoplasma asteris" associated with black locust witches' broom in Korea. J. Kor. For. Soc. 96:737-741.

Han, S. and Cha, B. 2001. Phylogenetic relationship of tree phytoplasmas in Korea. Kor. J. Mycoplasmol. 12:38-47.

Han, S. and Cha, B. 2002. Genetic similarity between jujube witches' broom and mulberry dwarf phytoplasmas transmitted by Hishimonus sellatus Uhler. Plant Pathol. J. 18:98-101.

Han, S., Kim, Y. H., So, I. Y. and Chai, J. K. 1997. Association of phytoplasma with chestnut (Castanea crenata Sieb. et Zucc.) little leaf disease in Korea. Kor. J. Mycoplasmol. 8:48-54.

Hogenhout, S. A., Oshima, K., Ammar, E. D., Kakizawa, S., Kingdom, H. N. and Namba, S. 2008. Phytoplasmas: bacteria that manipulate plants and insects. Mol. Plant Pathol. 9:403423.

Jung, H. Y. 2003. Phylogenetic classification of phytoplasmas that occur in Korea. Ph.D. Thesis. The University of Tokyo. Japan. $107 \mathrm{pp}$.

Jung, H. Y. and Lee, J. T. 2004. First report of Candidatus Phytoplasma pruni associated with yellow dwarf disease of Aster glehni var. hondoensis. Kor. J. Mycoplasmol. 15:35-41.

Jung, H. Y., Chang, M. U., Lee, J. T. and Namba, S. 2006. Detection of "Candidatus Phytoplasma asteris" associated with henon bamboo witches' broom in Korea. J. Gen. Plant Pathol. 72:261-263.

Jung, H. Y., Hahm, Y. I., Lee, J. T., Hibi, T. and Namba, S. $2003 \mathrm{a}$. Characterization of a phytoplasma associated with witches' broom disease of potatoes in Korea. J. Gen. Plant Pathol. 69:87-89.

Jung, H. Y., Sawayanagi, T., Kakizawa, S., Nishigawa, H., Miyata, S., Oshima, K., Ugaki, M., Lee, J. T., Hibi, T. and Namba, S. 2002a. 'Candidatus Phytoplasma castaneae', a novel phytoplasma taxon associated with chestnut witches' broom disease. Int. J. Syst. Evol. Microbiol. 52:1543-1549.

Jung, H. Y., Sawayanagi, T., Kakizawa, S., Nishigawa, H., Wei, W., Oshima, K., Miyata, S., Ugaki, M., Hibi, T. and Namba, S. 2003b. 'Candidatus Phytoplasma ziziphi', a novel phytoplasma taxon associated with jujube witches'-broom disease. Int. J. Syst. Evol. Microbiol. 53:1037-1041.

Jung, H. Y., Woo, T. H., Hibi, T., Namba, S. and Lee, J. T. 2002 b. Phylogenetic and taxonomic status of the phytoplasma associated with water dropwort (Oenanthe javanica DC) disease in Korea and Japan. Plant Pathol. J. 18:109-114.

Jung, H. Y., Yae, M. C., Lee, J. T., Hibi, T. and Namba, S. $2003 c$. Aster yellows subgroup (Candidatus Phytoplasma sp. AY 16S-group, AY-sg) phytoplasma associated with porcelain vine showing witches' broom symptoms in South Korea. $J$. Gen. Plant Pathol. 69:208-209. 
Kamala-Kannan, S., Han, S., Lee, K. J., Velmurugan, P., Lee, Y. H., Chae, J. C., Lee, Y. S., Lee, J. Y. and Oh, B. T. 2011. Association of elm yellows subgroup 16SrV-B phytoplasma with a disease of Hovenia dulcis. J. Phytopathol. 159:171-174.

Kamala-Kannan, S., Park, S. M., Oh, B. T., Kim, H. M. and Lee, K. J. 2010. First report of aster yellows phytoplasma in goldenrain tree (Koelreuteria paniculata) in Korea. J. Phytopathol. 158:197-199.

Kim, Y. H. 1980. Studies on mycoplasma-like organism associated with witches' broom of Rhus javanica (I). J. Kor. For. Soc. 47:1-15.

Kim, Y. H. and Jung, H. Y. 2007. Candidatus Phytoplasma trifolii associated with witches' broom of Lespedeza cyrtobotrya M. Plant Pathol. J. 23:106-108.

Kim, Y. H., Win, N. K. K., Back, C. G., Yea, M. C., Yim, K. O. and Jung, H. Y. 2011. Multiplex PCR assay for simultaneous detection of Korean quarantine phytoplasmas. Plant Pathol. J. 27:367-371.

Kube, M., Schneider, B., Kuhl, H., Dandekar, T., Heitmann, K., Migdoll, A. M., Reinhardt, R. and Seemüller, E. 2008. The linear chromosome of the plant-pathogenic mycoplasma 'Candidatus Phytoplasma mali'. BMC Genomics 9:306.

La, Y. J. and Bak, W. C. 1994. Insect transmission of paulownia witches'-broom mycoplasma-like organism to periwinkle plant by tobacco leaf bug, Cyrtopeltis tenuis Reuter. Korean J. Plant Pathol. 10:211-214.

La, Y. J., Park, W. C., Park, W. M. and Lee, Y. S. 1984. Electrophoresis of proteins in the tissues from witches' broom infected jujube tree. J. Kor. For. Soc. 66:79-81.

La, Y. J. and Woo, K. S. 1980. Transmission of jujube witches' broom mycoplasma by the leaf hopper Hishimonus sellatus Uhler. J. Kor. For. Soc. 48:29-39.

Lapage, S. P., Sneath, P. H. A., Lessel, E. F., Skerman, V. B. D., Seeliger, H. P. R. and Clark, W. A. 1992. International code of nomenclature of bacteria: bacteriological code. 1990 Revision. American Society for Microbiology, Washington, D.C. 189pp.

Lee, I. M., Hammond, R. W., Davis, R. E. and Gundersen, D. E. 1993. Universal amplification and analysis of pathogen $16 \mathrm{~S}$ rDNA for classification and identification of mycoplasma-like organisms. Phytopathology 83:834-842.

Lee, J. T. 1994. Vector and outbreak of cnidium witches'-broom infected by mycoplasma-like organism (MLO). Kor. J. Mycoplasmol. 5:60-68.

Lee, J. T. 2004. Phytoplasmal diseases in Korea. National Institute of Agricultural Science and Technology, Suwan, Korea. 226pp.

Lee, J. T. and Kim, E. H. 1994. Occurrence of witches' broom from Syringa dilatata, Quercus acutissima, Prunus salicina and Cryptotaenia japonica. Korean J. Mycoplasmol. 5:81.

Lee, J. T., Kim, E. H., Yea, M. C. and Kwon, O. Y. 1996. Detection of phytoplasmas from Paulownia tomentosa, Syringa vulgaris and Solidago vir-aurea var. gigantea using polymerase chain reaction (PCR) and their relationships. Kor. J. Plant Pathol. 12:191-196.

Lee, J. T., Kwak, W. J. and Kim, J. K. 2002. Occurrence of udo
(Aralia cordata) yellow dwarf caused by phytoplasma in Korea. Kor. J. Mycoplasmol. 13:55.

Lee, J. T. and Yea, M. C. 1992. On occurrence of witches' brooms of Vitis spp., Rubus crataegifolus and some medicinal plants in Korea. Korean J. Mycoplasmol. 3:64.

Lee, S., Han, S. and Cha, B. 2009. Mixed infection of 16 S rDNA I and $\mathrm{V}$ groups of phytoplasma in a single jujube tree. Plant Pathol. J. 25:21-25.

Murray, R. G. E. and Schleifer, K. H. 1994. Taxonomic notes: a proposal for recording the properties of putative taxa of prokaryotes. Int. J. Syst. Bacteriol. 44:174-176.

Namba, S. 2011. Phytoplasmas: a century of pioneering research. J. Gen. Plant Pathol. 77:345-349.

Namba, S., Kato, S., Iwanami, S., Oyaizu, H., Shiozawa, H. and Tsuchizaki, T. 1993. Detection and differentiation of plantpathogenic mycoplasmalike organisms using polymerase chain reaction. Phytopathology 83:786-791.

Oshima, K., Kakizawa, S., Nishigawa, H., Jung, H. Y., Wei, W., Suzuki, S, Arashida, R., Nakata, D., Miyata, S., Ugaki, M. and Namba, S. 2004. Reductive evolution suggested from the complete genome sequence of a plant-pathogenic phytoplasma. Nat. Genet. 36:27-29.

So, I. Y. 1973. Studies on the mycoplasmic witches'-broom of sweet potato in Korea. (I) Symptoms and pathogen. Korean J. Microbiol. 11:19-30.

Tamura, K., Dudley, J., Nei, M. and Kumar, S. 2007. MEGA4: molecular evolutionary genetics analysis (MEGA) software version 4.0. Mol. Biol. Evol. 24:1596-1599.

Tran-Nguyen, L. T. T., Kube, M., Schneider, B., Reinhardt, R. and Gibb, K. S. 2008. Comparative genome analysis of "Candidatus Phytoplasma australiense" (subgroup tuf-Australia I; $r p$-A) and "Ca. Phytoplasma asteris" strains OY-M and AY-WB. J. Bacteriol. 190:3979-3991.

Win, N. K. K., Lee, Y. H., Kim, Y. H., Back, C. G., Chung, H. and Jung, H. Y. 2012. Reclassification of aster yellows group phytoplasmas in Korea. J. Gen. Plant Pathol. DOI:10-1007/ s10327-012-0387-5.

Woo, T. H., Yae, M. C. and Lee, J. T. 2001. Occurrence of witches' broom disease and detection of the phytoplasma from water dropwort (Oenanthe javanica DC.) in Korea. Kor. J. Mycoplasmol. 12:48-60.

Yea, M. C. and Lee, J. T. 1997. Detection of phytoplasmas in Bupleurum falcantum and Crataegus pinnatifida. Kor. J. Mycoplasmol. 8:90.

Yea, M. C. and Lee, J. T. 2002. Occurrence of witches' broom disease of angustata-ash (Fraxinus sieboldiana var. angustata Blume) caused by phytoplasma in Korea. Kor. J. Mycoplasmol. 13:53.

Yeo, W. H., Bak, W. C., Lee, J. H., Koh, M. G., Yi, C. K. and Kim, Y. H. 1994. Transmission of paulownia witches' broom mycoplasma-like organisms to periwinkle by a leafhopper, Empoasca sp.. J. Kor. For. Soc. 83:1-5.

Yi, C. K. and La, Y. J. 1973. Mycoplasma-like bodies found in the phloem elements of jujube trees infected with witches' broom disease. Res. Rep. For. Res. Inst. 20:111-114. 